\title{
Land Evaluation of Old Irrigated Soils in North Delta Region (Rewena Canal Area) at Kafr El Sheikh Governorate
}

\author{
Zamil,B.A; M.A.Abd Allah, G.M.Abd El-Salam and M.I .El-Shahawy 1
}

\begin{abstract}
Quantified land evaluation of soils at Kafr El-sheikh governorate in north delta was carried out. These soils represent the area of Rewena canal and located between Kafr El-Sheikh and Sidi Salem district. Land capability and suitability for different crops was made through defining and determining soil physical, chemical properties and environmental properties as well as nutrients status. The quality of irrigation water was also determined as well as climatic data.
\end{abstract}

ASLE program (Applied System of Land Evaluation) was used for calculating land capability and there suitability for different vegetable, crops and fruits with a total of 28 plants.

Results indicate that the studied area was classified into two land capability classes: class 2 (good) and class 3 (faire).

Limitation factors for land capability were the relatively low soil permeability, shallow ground water table in some parts, the relative increment of soil salinity in others, as well as ground water salinity and low levels of soil organic matter and nutrients especially NPK.

Concerning land suitability, different crops can be grown in these soils except pepper, Olive, Fig and Peanut, while the suitable crops could be arranged by preference as: Barely $>$ Wheat $>$ Sugar beet $>$ Sunflower $>$ Cotton $>$ Rice.

\section{INTRODUCTION}

Agricultural production plays an important role in the Egyptian economy. It is considered as the source of national income and the way of life for a sizable part of the population. The agricultural sector in Egypt absorbs $38.2 \%$ of the labor force and able to absorb more. Egyptian Agricultural lands occupy about $4 \%$ (about 8.3 million Feddans) of Egypt area (FAO, 2001). Egypt is now facing a major challenge of how to increase the rate of growth in agriculture production to generate and meet its future food requirements to cope a very high annual rate of population increasing $(2.3 \%)$. The national strategy of Egypt aims to adding about 4.32 million Feddens of new land reclamation until year 2017 in different region, based on land suitability and water resources availability (GARPAD) 1997.

Land evaluation is a term used to describe the process of collating and interpreting basic inventories of soil, vegetation cover, environmental condition, climatic

\footnotetext{
${ }^{1}$ Soils, Water and Env. Res. Inst., Argric. Res. Center, Egypt.
} Received May 19, 2009, Accepted June 8, 2009 status and many other aspects of land in order to identify and compare land use alternative.

Riquier et al (1970) proposed the parametric method of land evaluation and claimed that limitations, as negative and complex concepts in both present and future capability, are better expressed in terms of productivity.

Sys and Verheye (1972) suggested the calculation of a productivity index as an indication of land capability according to multiplication method. Five main groups of parameters were included namely, soil physical, and soil chemical, topographic, soil fertility and irrigation water parameters.

According to FAO (1976), land evaluation is the prediction of land performance overtime under specific uses. Sys (1979) stated that land evaluation is an opinion, an assessment, a careful judgment. The land evaluation objective is guide wisely the present management and plan the future and best land use among alternatives.

Abd El-Motteleb and Hussein (1985) (Arabic) considered that soil characteristics and environmental conditions are the main factor of land productivity and land classification. In this system, six soil classes were introduced, based on both soil properties and environmental conditions.

Marie et al (1987) proposed a computer program for land evaluation system (LE) based on that of Abd El-Motteleb and Hussien (1985). This system was modified by EL-Fayoumy (1989) to include soil fertility and irrigation water factor. The last form of this system was developed as a new edition (ASLE) (Morsy, 1994) by adding land suitability to different crops based on land properties as well as climatic data. Each factor was described as an index value to give its statues in the percentage form.

Ismail et al (2001), by using (ASLE), sited that Samoul area (was part of Nile Delta Region) could be classified into two good and moderate capability classes. Where Burg El Arab and El-Shahama (in the western desert) area was Moderate and Marginal capability classes. He also added that the main limitations were low and high soil permeability, low percentage of clay, shallow water table, soil salinity, soil structure, low soil organic matter and nutrients. 
Naser Eldin (2001) in his study on Kafr El-Sheikh Governorate soil found that, land capability classes were Excellent, good, faire and poor and the main limitations were shallow water table, drainage system and nutrients.

Fayed (2003) evaluated the land capability of ElBostan region West Nile Delta. He found that, the studied area classified into two land capability (Moderate and Marginal). He also added that, the main limiting soil factors in the studied soils were soil texture, ESP, salinity and calcium carbonate content.

Higab (2005) evaluate some soils of south ElBorolus Lake area. He found that the capability index for these soils area $\left(\mathrm{S}_{2}\right)$ good soil, $\left(\mathrm{S}_{3}\right)$ fair soil and $\left(\mathrm{N}_{1}\right)$ non-agriculture soils.

\section{MATERIAL AND METHODS}

The area is located at North Delta (Fig.1), the elevation was varied between $2 \mathrm{~m}$ and $6 \mathrm{~m}$ a.s.1., at Kafr El-Sheikh Governorate, beside Rewena Canal. It is bounded by Sidi Salem sector (El-Masharqa village) from the North to Rewena village from the South and Nashart Drainage from the West to drainage no. 7 from the East (Fig. 2).

These areas were irrigated by Fresh water from Rewena canal and served by tile drainage system. The location of the studied area is shown in map (Fig.2).

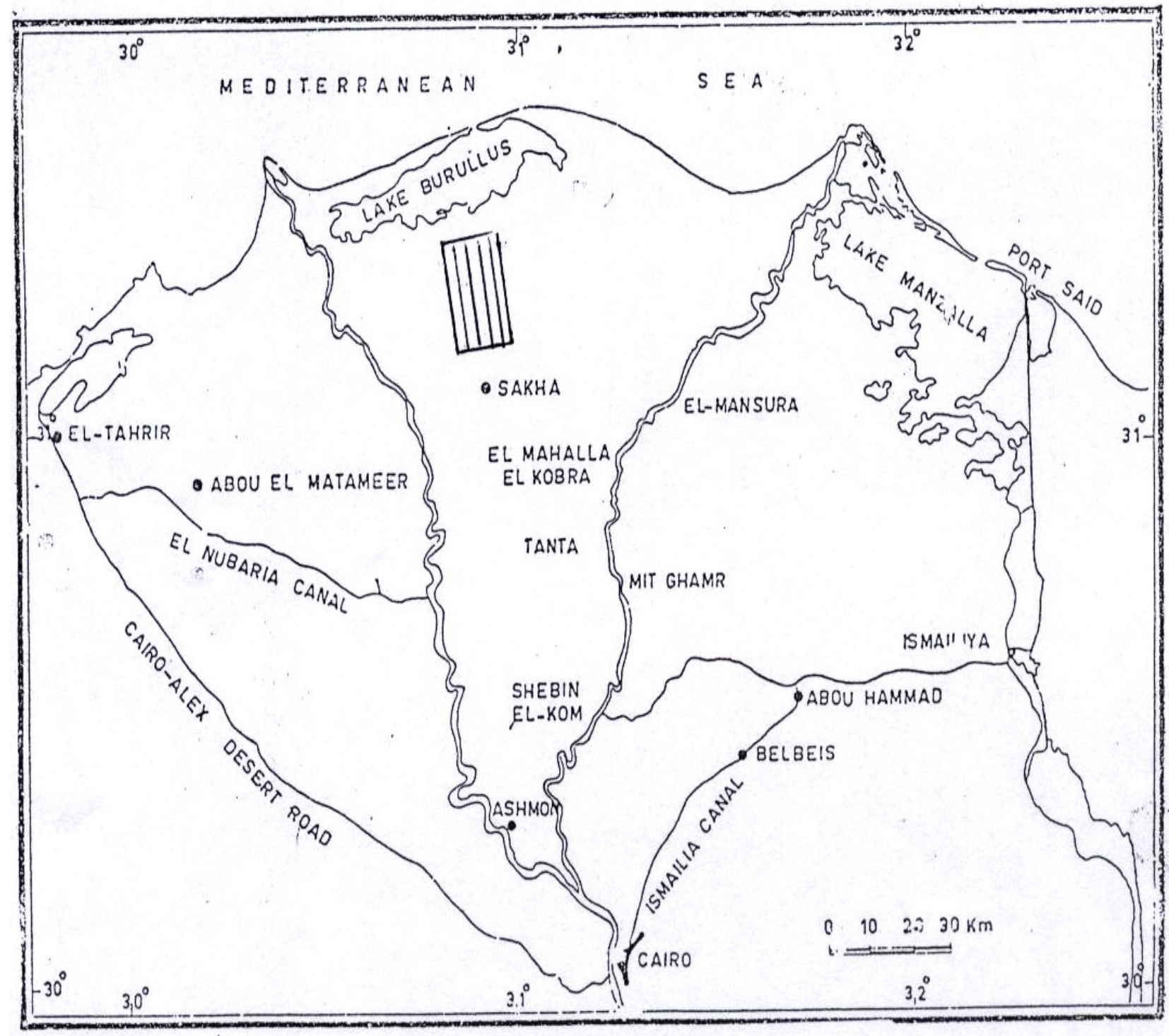

Fig.1. Location of the studied area 


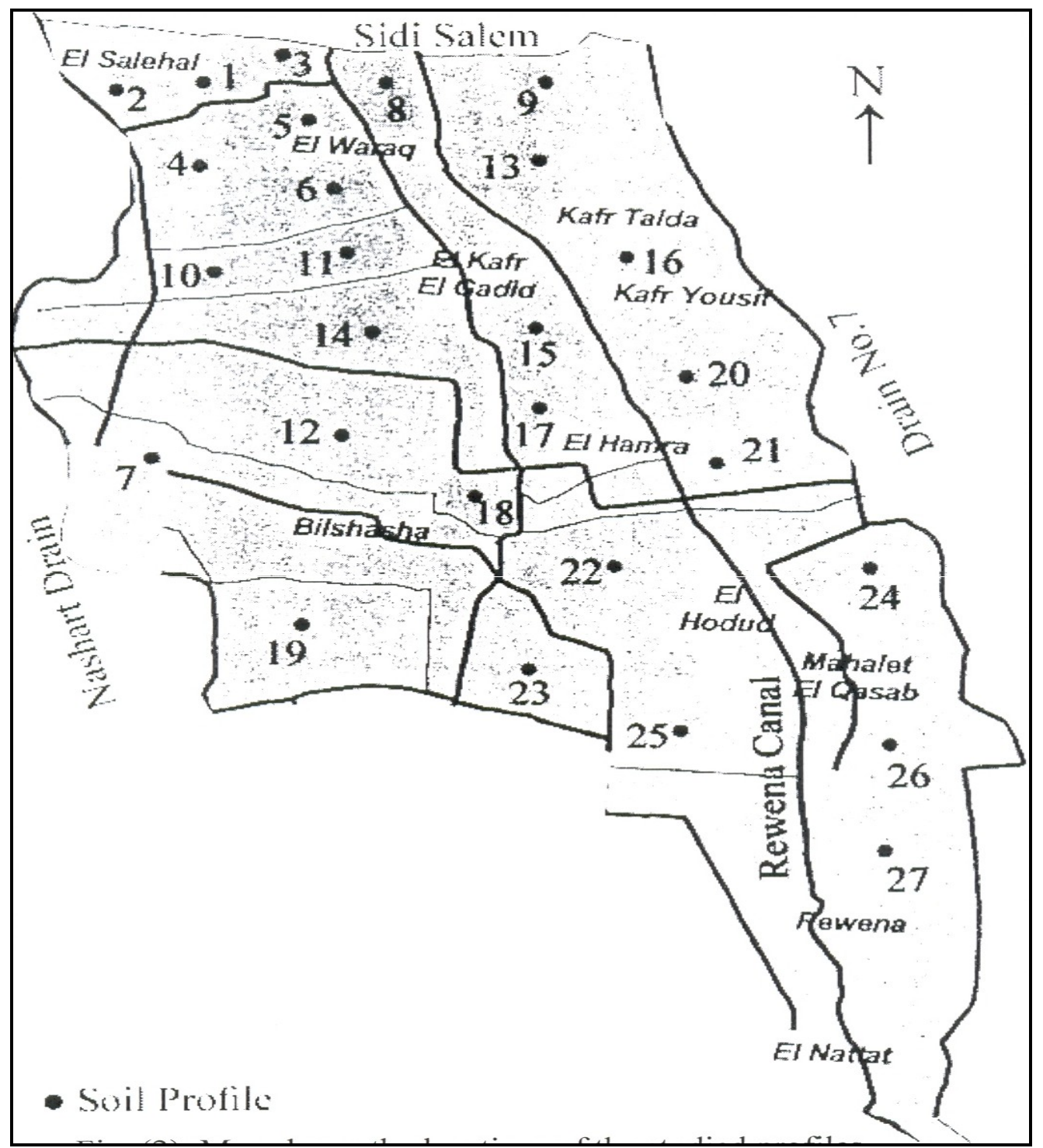

Fig. 2. Map shows the locations of the studied profiles

Field work and laboratory analysis:

Twenty seven soil profiles were selected as old lands from Rewena command area at kafr El-Sheikh governorate and sited using GPS. Soil samples were collected from different layers according to morphological variations or equal distances for homogeneous profiles and were subjected to different physical and chemical analysis as irrigation and ground water samples.

\section{Samples analysis:}

$\mathrm{EC}(\mathrm{dS} / \mathrm{m}), \mathrm{PH}, \mathrm{OM} \%$ and $\mathrm{CaCO} 3 \%$ according to Jackson, (1973).

ESP was calculated according to the formula:

$\mathrm{ESP}=100(-0.0126+0.01475 \mathrm{SAR}) \quad,($ Richard, 1954).

$$
1+(-0.0126+0.01475 \text { SAR })
$$

CEC according to Klute (1986).

Available N and K, according to Cottenie et al (1982).

Available P, according to Olsen et al (1954).

Mechanical analysis, according to Piper (1950).

Hydraulic conductivity (as Ks) by auger hole method according to Van Beers (1970).

Structure Factor was calculated according to the following formula:

$$
\mathrm{SF}=\left\{1-\frac{\% \text { clay in aggregation analysis }}{\% \text { clay in mechanical analysis }}\right\} \times 100
$$

Available water (A.W.) calculated according to the formula: 
Available water = filed capacity - wilting point, according to Kulte (1986).

\section{Land evaluation}

Land evaluation and quantified recommendations for soil improvement were implemented using "applied system for land evaluation (ASLE) ". This system calculates the land evaluation as a percentage value based on four main factors; soil properties, irrigation water quality, soil fertility and environmental conditions as well as climatic data. The final index of land evaluation (F.I.L.E) was calculated as:

$$
4
$$

F.I.L.E $=\longrightarrow$ Ismail et al (1994)

$$
\frac{1}{\text { S.I }}+\frac{1}{\text { W.I }}+\frac{1+\frac{1}{\text { F.I }}}{\text { E.I }}
$$

Where: S.I: the soil index.
F.I: the soil fertility index.

E.I: the environmental index.

\section{RESULTS AND DISCUSSION}

\section{Soil characteristics:}

\section{Soil physical properties:}

Data in Tables (1) revealed that, clay content ranged between $21.80 \%$ to $63.10 \%$. The soil depth is moderate and ranged from $60 \mathrm{~cm}$ to $120 \mathrm{~cm}$. The soil structure factor ranged from $18.60 \%$ to $44.60 \%$, while the hydraulic conductivity is low and ranged from 0.13 $\mathrm{cm} / \mathrm{h}$ to $4.83 \mathrm{~cm} \mathrm{/} \mathrm{h}$. These low values may be attributed to the decrease of organic matter content and higher ESP and SAR values (Madkour et al, 1999). The available water varied from $13.62 \%$ to $22.34 \%$ and it depends on clay and organic matter.

W.I: the irrigation water index.

\begin{tabular}{|c|c|c|c|c|c|c|c|}
\hline P. no. & Depth cm & Clay \% & S.F $\%$ & AW \% & $\mathrm{K}_{\mathrm{s}} \quad \mathrm{cm} / \mathrm{h}$ & Profile depth $\mathrm{cm}$ & Slop \% \\
\hline \multirow{3}{*}{1} & $0-25$ & 57.5 & 33.8 & 20.06 & & & \\
\hline & $25-65$ & 60.1 & 40.6 & 9.58 & 1.49 & 90 & 1.50 \\
\hline & $65-100$ & 58.2 & 39.5 & & & & \\
\hline \multirow{3}{*}{2} & $0-20$ & 56.2 & 32.8 & 18.14 & & & \\
\hline & $20-60$ & 57.2 & 32.6 & 16.18 & 4.83 & 120 & 1.50 \\
\hline & $60-100$ & 55.3 & 31.7 & & & & \\
\hline \multirow{3}{*}{3} & $0-25$ & 46.2 & 30.8 & 19.43 & & & \\
\hline & $25-70$ & 48.8 & 31.2 & 16.73 & 0.73 & 100 & 1.50 \\
\hline & $70-100$ & 44.2 & 33.6 & & & & \\
\hline \multirow{3}{*}{4} & $0-20$ & 51.7 & 34.5 & 18.45 & & & \\
\hline & $20-50$ & 55.9 & 36.8 & 17.6 & 0.58 & 90 & 1.50 \\
\hline & $50-100$ & 52.5 & 31.8 & & & & \\
\hline \multirow{3}{*}{5} & $0-25$ & 55.1 & 35.8 & 18.05 & & & \\
\hline & $25-65$ & 57.6 & 35.2 & 18.41 & 3.67 & 90 & 1.50 \\
\hline & $65-100$ & 36.2 & 22.4 & & & & \\
\hline \multirow{3}{*}{6} & $0-25$ & 51.5 & 35.8 & 18.35 & & & \\
\hline & $25-70$ & 49.5 & 31.4 & 18.15 & 3.84 & 100 & 1.60 \\
\hline & $70-100$ & 45.1 & 32.2 & & & & \\
\hline \multirow{3}{*}{7} & $0-20$ & 55.3 & 35.1 & 20.41 & & & \\
\hline & $20-60$ & 59.4 & 37.6 & 18.35 & 1.23 & 90 & 1.60 \\
\hline & $60-100$ & 56.5 & 36.2 & & & & \\
\hline \multirow{3}{*}{8} & $0-20$ & 48.6 & 32.4 & 19.94 & & & \\
\hline & $20-55$ & 54 & 35.2 & 17.56 & 1.25 & 100 & 1.60 \\
\hline & $55-100$ & 53.7 & 34.2 & & & & \\
\hline \multirow{3}{*}{9} & $0-25$ & 56 & 34.2 & 22.33 & & & \\
\hline & $25-65$ & 52.6 & 31.9 & 17.86 & 1.24 & 90 & 1.60 \\
\hline & $65-100$ & 25.5 & 18.6 & & & & \\
\hline \multirow{3}{*}{10} & $0-20$ & 60.60 & 44.60 & 22.26 & & & \\
\hline & $20-60$ & 58.70 & 33.80 & 18.08 & 1.21 & 90 & 1.30 \\
\hline & $60-100$ & 60.50 & 40.20 & & & & \\
\hline \multirow{2}{*}{11} & $0-20$ & 52.60 & 38.60 & 13.62 & 043 & 80 & 130 \\
\hline & $20-55$ & 56.20 & 39.00 & 17.50 & 0.43 & 80 & 1.50 \\
\hline
\end{tabular}

Table 1. Physical properties of the studied soil of studied profiles 
55-100

54.30

35.60

Table 1. Cont

\begin{tabular}{|c|c|c|c|c|c|c|c|}
\hline P. no. & Depth cm & Clay \% & S.F $\%$ & AW \% & $\mathrm{K}_{\mathrm{s}} \quad \mathrm{cm} / \mathrm{h}$ & Profile depth $\mathrm{cm}$ & Slope \% \\
\hline \multirow{3}{*}{12} & $0-20$ & 57.30 & 36.80 & 20.96 & & & \\
\hline & $20-55$ & 63.10 & 42.20 & 21.43 & 0.13 & 90 & 1.50 \\
\hline & $55-100$ & 59.20 & 40.80 & & & & \\
\hline \multirow{3}{*}{13} & $0-30$ & 59.20 & 40.10 & 17.66 & & & \\
\hline & $30-60$ & 56.60 & 36.20 & 15.73 & 0.49 & 60 & 1.50 \\
\hline & $60-100$ & 21.80 & 18.40 & & & & \\
\hline \multirow{3}{*}{14} & $0-20$ & 46.70 & 30.20 & 22.34 & & & \\
\hline & $20-60$ & 48.30 & 32.60 & 21.12 & 1.30 & 100 & 1.60 \\
\hline & $60-90$ & 48.50 & 33.10 & & & & \\
\hline \multirow{3}{*}{15} & $0-20$ & 59.10 & 37.10 & 18.40 & & & \\
\hline & $20-50$ & 54.00 & 36.40 & 21.99 & 1.68 & 100 & 1.60 \\
\hline & $50-90$ & 22.60 & 16.80 & & & & \\
\hline \multirow{3}{*}{16} & $0-30$ & 44.70 & 33.40 & 18.32 & & & \\
\hline & $30-60$ & 28.60 & 18.20 & 17.19 & 5.87 & 90 & 1.50 \\
\hline & $60-100$ & 20.50 & 16.80 & & & & \\
\hline \multirow{3}{*}{17} & $0-20$ & 59.30 & 39.20 & 21.27 & & & \\
\hline & $20-60$ & 62.90 & 42.80 & 17.50 & 1.52 & 100 & 1.60 \\
\hline & $60-100$ & 60.60 & 40.40 & & & & \\
\hline \multirow{3}{*}{18} & $0-35$ & 49.10 & 33.30 & 17.42 & & & \\
\hline & $35-75$ & 57.40 & 36.70 & 17.64 & 3.57 & 100 & 1.60 \\
\hline & $75-100$ & 54.70 & 36.80 & & & & \\
\hline \multirow{3}{*}{19} & $0-20$ & 57.30 & 38.00 & 21.03 & & & \\
\hline & $20-50$ & 57.80 & 38.20 & 21.11 & 0.69 & 100 & 1.60 \\
\hline & $50-100$ & 56.90 & 36.40 & & & & \\
\hline \multirow{3}{*}{20} & $0-20$ & 53.30 & 33.10 & 13.72 & & & \\
\hline & $20-60$ & 41.60 & 39.20 & 15.35 & 5.60 & 90 & 1.50 \\
\hline & $60-90$ & 47.20 & 40.20 & & & & \\
\hline \multirow{3}{*}{21} & $0-20$ & 57.80 & 41.80 & 20.35 & & & \\
\hline & $20-50$ & 54.00 & 38.20 & 21.34 & 0.59 & 80 & 1.60 \\
\hline & $50-80$ & 55.90 & 36.90 & & & & \\
\hline \multirow{3}{*}{22} & $0-20$ & 56.90 & 36.20 & 20.30 & & & \\
\hline & $20-55$ & 60.40 & 44.60 & 19.86 & 2.00 & 100 & 1.50 \\
\hline & $55-100$ & 59.20 & 41.20 & & & & \\
\hline \multirow{3}{*}{23} & $0-15$ & 57.30 & 32.80 & 18.43 & & & \\
\hline & $15-45$ & 62.10 & 40.70 & 19.34 & 0.35 & 100 & 1.50 \\
\hline & $45-100$ & 55.20 & 36.20 & & & & \\
\hline \multirow{3}{*}{24} & $0-15$ & 51.40 & 29.90 & 17.84 & & & \\
\hline & $15-40$ & 52.80 & 31.20 & 18.10 & 3.19 & 80 & 1.60 \\
\hline & $40-80$ & 52.50 & 31.60 & & & & \\
\hline \multirow{3}{*}{25} & $0-20$ & 59.20 & 40.50 & 17.21 & & & \\
\hline & $20-50$ & 56.60 & 32.60 & 17.99 & 2.32 & 80 & 1.60 \\
\hline & $50-80$ & 21.80 & 21.20 & & & & \\
\hline \multirow{3}{*}{26} & $0-15$ & 44.80 & 36.10 & 16.51 & & & \\
\hline & $15-50$ & 48.20 & 32.60 & 18.45 & 1.92 & 100 & 1.60 \\
\hline & $50-100$ & 46.90 & 30.20 & & & & \\
\hline \multirow{3}{*}{27} & $0-15$ & 52.80 & 32.90 & 15.95 & & & \\
\hline & $15-60$ & 54.60 & 30.20 & 15.56 & 2.71 & 100 & 1.60 \\
\hline & $60-100$ & 23.70 & 18.90 & & & & \\
\hline
\end{tabular}


Soil chemical properties:

Data in Tables (2) showed that, EC values varied from 0.96 to $11.9 \mathrm{dS} / \mathrm{m}$. The CEC values ranged from

Table 2. Chemical properties of the studied soil

\begin{tabular}{|c|c|c|c|c|c|c|c|}
\hline P. no. & $\begin{array}{c}\text { Depth } \\
(\mathrm{cm})\end{array}$ & pH & $\begin{array}{l}\mathrm{ECe} \\
\mathrm{ds} / \mathrm{m}\end{array}$ & $\begin{array}{c}\text { GWS } \\
\text { ppm }\end{array}$ & $\begin{array}{c}\text { CEC } \\
\text { meg/100 }\end{array}$ & ESP\% & $\mathrm{CaCO}_{3} \%$ \\
\hline \multirow{3}{*}{1} & $0-25$ & 8.20 & 8.13 & \multirow{3}{*}{7280} & 66.86 & 13.05 & 1.90 \\
\hline & $25-65$ & 8.30 & 2.72 & & 65.56 & 15.15 & 1.70 \\
\hline & $65-100$ & 8.35 & 4.52 & & 62.46 & 20.1 & 1.70 \\
\hline \multirow{3}{*}{2} & $0-20$ & 8.10 & 4.28 & \multirow{3}{*}{6412} & 66.7 & 14.75 & 1.60 \\
\hline & $20-60$ & 7.71 & 4.18 & & 73.88 & 10.25 & 1.40 \\
\hline & $60-100$ & 7.90 & 1.48 & & 53.84 & 13.6 & 1.30 \\
\hline \multirow{3}{*}{3} & $0-25$ & 8.00 & 2.38 & \multirow{3}{*}{7680} & 43.12 & 11.6 & 2.70 \\
\hline & $25-70$ & 8.40 & 3.18 & & 52.12 & 18.5 & 2.90 \\
\hline & $70-100$ & 8.50 & 11.1 & & 42.9 & 19.11 & 2.80 \\
\hline \multirow{3}{*}{4} & $0-20$ & 7.50 & 3.85 & \multirow{3}{*}{8000} & 62.33 & 10.43 & 1.50 \\
\hline & $20-50$ & 8.10 & 5.99 & & 44.9 & 16.3 & 1.50 \\
\hline & $50-100$ & 7.40 & 11.9 & & 46.16 & 9.84 & 1.30 \\
\hline \multirow{3}{*}{5} & $0-25$ & 8.60 & 4.84 & \multirow{3}{*}{2560} & 48.3 & 16.4 & 1.50 \\
\hline & $25-65$ & 8.10 & 1.26 & & 49.24 & 7.35 & 1.40 \\
\hline & $65-100$ & 8.00 & 1.53 & & 44.44 & 5.73 & 1.40 \\
\hline \multirow{3}{*}{6} & $0-25$ & 7.10 & 3.68 & \multirow{3}{*}{3200} & 66.66 & 8.1 & 1.80 \\
\hline & $25-70$ & 7.30 & 2.41 & & 69.3 & 12.24 & 1.50 \\
\hline & $70-100$ & 7.30 & 2.33 & & 52.52 & 12.38 & 1.50 \\
\hline \multirow{3}{*}{7} & $0-20$ & 7.10 & 9.8 & \multirow{3}{*}{8320} & 71.9 & 9.04 & 1.70 \\
\hline & $20-60$ & 7.20 & 9.6 & & 69.68 & 11.94 & 1.70 \\
\hline & $60-100$ & 8.10 & 9.88 & & 66.78 & 15.99 & 1.50 \\
\hline \multirow{3}{*}{8} & $0-20$ & 7.70 & 1.91 & \multirow{3}{*}{3200} & 42.24 & 7.1 & 1.50 \\
\hline & $20-55$ & 7.70 & 1.91 & & 59.51 & 6.91 & 1.50 \\
\hline & $55-100$ & 8.10 & 3.13 & & 55.76 & 17.93 & 1.40 \\
\hline \multirow{3}{*}{9} & $0-25$ & 8.00 & 8.16 & \multirow{3}{*}{5760} & 64.1 & 13.96 & 1.90 \\
\hline & $25-65$ & 7.90 & 3.87 & & 63.13 & 11.15 & 1.70 \\
\hline & $65-100$ & 7.90 & 7.07 & & 33.4 & 15.9 & 1.50 \\
\hline \multirow{3}{*}{10} & $0-20$ & 8.30 & 9.91 & \multirow{3}{*}{2650} & 70.71 & 16.30 & 1.90 \\
\hline & $20-60$ & 7.90 & 2.01 & & 30.31 & 8.73 & 1.70 \\
\hline & $60-100$ & 8.40 & 2.65 & & 79.55 & 16.81 & 1.50 \\
\hline \multirow{3}{*}{11} & $0-20$ & 7.91 & 2.84 & \multirow{3}{*}{4480} & 52.06 & 8.29 & 1.60 \\
\hline & $20-55$ & 7.60 & 4.50 & & 53.86 & 14.07 & 1.40 \\
\hline & $55-100$ & 8.10 & 5.14 & & 55.09 & 13.50 & 1.40 \\
\hline \multirow{3}{*}{12} & $0-20$ & 7.60 & 5.36 & \multirow{3}{*}{6400} & 79.80 & 12.47 & 1.70 \\
\hline & $20-55$ & 7.60 & 4.01 & & 78.44 & 13.51 & 1.50 \\
\hline & $55-100$ & 8.20 & 7.51 & & 69.22 & 17.05 & 1.50 \\
\hline & $0-30$ & 7.20 & 5.66 & & 67.34 & 8.43 & 2.10 \\
\hline 13 & $30-60$ & 7.20 & 1.86 & 5120 & 46.92 & 6.64 & 2.30 \\
\hline & $60-100$ & 8.40 & 3.95 & & 53.06 & 14.78 & 1.90 \\
\hline & $0-20$ & 7.01 & 1.44 & & 64.43 & 6.09 & 1.70 \\
\hline 14 & $20-60$ & 7.01 & 1.05 & 3520 & 67.25 & 5.32 & 1.50 \\
\hline & $60-90$ & 8.40 & 2.56 & & 67.51 & 12.10 & 1.50 \\
\hline
\end{tabular}

33.09 to 88.86 meq./100 g soil. While ESP values ranged from 3.85 to $20.1 \%$; calcium carbonate content varied from $1.3 \%$ to $3.70 \%$. 
Table 2. Cont

\begin{tabular}{|c|c|c|c|c|c|c|c|}
\hline P. no. & $\begin{array}{c}\text { Depth } \\
(\mathrm{cm})\end{array}$ & pH & $\begin{array}{r}\text { ECe } \\
\text { ds/m }\end{array}$ & $\begin{array}{c}\text { GWS } \\
\text { ppm }\end{array}$ & $\begin{array}{c}\text { CEC } \\
\text { meg/100 }\end{array}$ & ESP\% & $\mathrm{CaCO}_{3} \%$ \\
\hline \multirow{3}{*}{15} & $0-20$ & 7.20 & 3.35 & \multirow{3}{*}{5120} & 75.01 & 6.10 & 1.70 \\
\hline & $0-50$ & 7.20 & 1.41 & & 75.02 & 5.40 & 1.70 \\
\hline & $50-90$ & 7.40 & 1.60 & & 34.10 & 7.20 & 1.50 \\
\hline \multirow{3}{*}{16} & $0-30$ & 7.60 & 3.21 & \multirow{3}{*}{5760} & 59.66 & 10.00 & 1.70 \\
\hline & $30-60$ & 7.90 & 4.56 & & 38.97 & 13.20 & 1.30 \\
\hline & $60-100$ & 7.90 & 5.32 & & 27.11 & 14.81 & 1.30 \\
\hline \multirow{3}{*}{17} & $0-20$ & 8.10 & 4.32 & \multirow{3}{*}{3840} & 76.49 & 13.70 & 1.80 \\
\hline & $20-60$ & 8.60 & 1.38 & & 88.86 & 10.20 & 1.90 \\
\hline & $60-100$ & 8.90 & 4.44 & & 72.75 & 17.30 & 1.50 \\
\hline \multirow{3}{*}{18} & $0-35$ & 7.40 & 1.88 & \multirow{3}{*}{1920} & 57.54 & 8.75 & 2.60 \\
\hline & $35-75$ & 7.01 & 1.56 & & 52.84 & 3.85 & 2.80 \\
\hline & $75-100$ & 7.40 & 1.32 & & 50.48 & 11.05 & 2.80 \\
\hline \multirow{3}{*}{19} & $0-20$ & 8.40 & 5.67 & \multirow{3}{*}{4480} & 64.80 & 11.94 & 3.70 \\
\hline & $20-50$ & 8.90 & 4.80 & & 68.86 & 13.54 & 3.50 \\
\hline & $50-100$ & 8.90 & 5.30 & & 69.29 & 13.15 & 3.10 \\
\hline \multirow{3}{*}{20} & $0-20$ & 7.40 & 5.25 & \multirow{3}{*}{2560} & 50.68 & 7.04 & 2.30 \\
\hline & $20-60$ & 8.10 & 3.10 & & 59.09 & 9.94 & 2.50 \\
\hline & $60-90$ & 8.40 & 1.41 & & 61.04 & 8.88 & 2.30 \\
\hline \multirow{3}{*}{21} & $0-20$ & 8.50 & 4.56 & \multirow{3}{*}{2560} & 62.33 & 10.43 & 2.30 \\
\hline & $20-50$ & 5.20 & 1.64 & & 65.14 & 7.84 & 2.50 \\
\hline & $50-80$ & 8.70 & 1.87 & & 67.34 & 8.94 & 2.30 \\
\hline \multirow{3}{*}{22} & $0-20$ & 8.01 & 1.23 & \multirow{3}{*}{1920} & 56.84 & 5.28 & 2.60 \\
\hline & $20-55$ & 8.11 & 0.96 & & 56.62 & 6.92 & 2.20 \\
\hline & $55-100$ & 8.36 & 1.52 & & 56.08 & 9.63 & 2.20 \\
\hline \multirow{3}{*}{23} & $0-15$ & 8.40 & 3.98 & \multirow{3}{*}{5760} & 53.32 & 10.30 & 3.60 \\
\hline & $15-45$ & 8.11 & 6.71 & & 56.44 & 14.39 & 3.70 \\
\hline & $45-100$ & 8.40 & 6.67 & & 43.52 & 13.25 & 3.50 \\
\hline \multirow{3}{*}{24} & $0-15$ & 8.21 & 2.05 & \multirow{3}{*}{2560} & 39.31 & 11.54 & 2.30 \\
\hline & $15-40$ & 8.36 & 1.61 & & 47.31 & 18.96 & 2.50 \\
\hline & $40-80$ & 8.49 & 1.65 & & 45.24 & 12.27 & 2.50 \\
\hline \multirow{3}{*}{25} & $0-20$ & 8.40 & 5.66 & \multirow{3}{*}{4480} & 46.92 & 8.94 & 2.10 \\
\hline & $20-50$ & 7.50 & 1.86 & & 43.06 & 6.64 & 2.30 \\
\hline & $50-80$ & 8.60 & 3.95 & & 40.60 & 14.78 & 1.90 \\
\hline \multirow{3}{*}{26} & $0-15$ & 8.02 & 1.06 & \multirow{3}{*}{2240} & 45.42 & 5.42 & 3.70 \\
\hline & $15-50$ & 8.10 & 1.08 & & 43.76 & 5.40 & 3.50 \\
\hline & $50-100$ & 8.10 & 1.23 & & 43.51 & 5.50 & 3.50 \\
\hline \multirow{3}{*}{27} & $0-15$ & 8.10 & 1.12 & & 39.10 & 5.58 & 2.40 \\
\hline & $15-60$ & 8.15 & 1.21 & 2240 & 33.09 & 5.32 & 2.40 \\
\hline & $60-100$ & 8.20 & 1.23 & & 42.84 & 6.00 & 1.50 \\
\hline
\end{tabular}

\section{GWS: Ground Water Salinity}

\section{Soil fertility:}

Data in Table (3) revealed that organic matter content is low, where it varied from $0.95 \%$ to $2.3 \%$ the decrease of OM content may be due to the increase of decomposition under high degree of temperature in arid and semi-arid region

\section{Land Capability Classification:}


Data in table (4) indicated that final index of land evaluation (F.I.L.E) ranges between $48.11 \%$ and 68.83 $\%$, so the area could be classified as $\mathrm{C}_{2}$ (good) and $\mathrm{C}_{3}$ (fair). Concerning land capability limitation data revealed that the most limiting factors are soil factor and fertility status.

Accordingly, the main limiting properties for land capability of Rewena canal area at Kafr El-sheikh governorate are ground water depth (GWD) and hydraulic conductivity (as $\mathrm{K}_{\mathrm{S}}$ ) as a physical soil properties, soil salinity (ECe) and ground water salinity
(GWS) as a chemical soil properties, soil organic matter content (OM\%) and available macro nutrients (NPK) as a soil fertility. However there are no limiting factors concerning neither environmental conditions or irrigation water quality.

\section{2-Land suitability for crops:}

The land suitability classes for crops were determined by matching land qualities, climatic data (Table 5) and requirement throughout the suggested computer model.

\section{Table 3. Soil fertility properties of Rewena area}

\begin{tabular}{|c|c|c|c|c|c|c|c|}
\hline \multirow{2}{*}{ P. no. } & \multirow{2}{*}{$\begin{array}{c}\text { Depth } \\
\text { (cm) }\end{array}$} & \multirow{2}{*}{ OM \% } & \multicolumn{3}{|c|}{ Available (ppm) } & \multicolumn{2}{|c|}{ Exch., meq/100g } \\
\hline & & & $\mathbf{N}$ & $\mathbf{P}$ & $\mathbf{K}^{+}$ & $\mathbf{C a}^{++}$ & $\mathrm{Mg}++$ \\
\hline \multirow{3}{*}{1} & $0-20$ & 2.30 & 15.80 & 3.69 & 140.40 & 36.00 & 22.10 \\
\hline & $20-65$ & 1.90 & 15.00 & 4.01 & 143.60 & 26.38 & 28.43 \\
\hline & $65-100$ & & & & & 21.98 & 27.37 \\
\hline \multirow{3}{*}{2} & $0-20$ & 2.05 & 28.30 & 6.34 & 370.50 & 43.00 & 12.46 \\
\hline & $20-60$ & 1.95 & 26.10 & 5.90 & 372.40 & 45.36 & 20.20 \\
\hline & $60-100$ & & & & & 26.38 & 19.82 \\
\hline \multirow{3}{*}{3} & $0-25$ & 1.25 & 8.20 & 1.63 & 163.80 & 22.87 & 14.77 \\
\hline & $25-70$ & 1.04 & 10.20 & 3.40 & 160.20 & 21.78 & 17.26 \\
\hline & $70-100$ & & & & & 22.87 & 12.23 \\
\hline \multirow{3}{*}{4} & $0-20$ & 1.74 & 80.40 & 1.26 & 128.70 & 24.70 & 25.18 \\
\hline & $20-50$ & 1.30 & 69.50 & 2.14 & 130.50 & 25.74 & 11.50 \\
\hline & $50-100$ & & & & & 31.68 & 9.84 \\
\hline \multirow{3}{*}{5} & $0-25$ & 1.90 & 83.60 & 2.15 & 163.80 & 22.40 & 17.42 \\
\hline & $25-65$ & 1.60 & 80.40 & 2.50 & 166.70 & 29.70 & 15.38 \\
\hline & $65-100$ & & & & & 24.28 & 17.26 \\
\hline \multirow{3}{*}{6} & $0-25$ & 2.04 & 29.70 & 1.10 & 226.20 & 41.76 & 18.26 \\
\hline & $25-70$ & 1.60 & 25.20 & 0.98 & 216.30 & 34.07 & 25.95 \\
\hline & $70-100$ & & & & & 27.48 & 17.80 \\
\hline \multirow{3}{*}{7} & $0-20$ & 1.45 & 74.60 & 4.03 & 261.30 & 35.64 & 29.04 \\
\hline & $20-60$ & 1.20 & 61.50 & 5.12 & 280.50 & 37.62 & 23.14 \\
\hline & $60-100$ & & & & & 30.34 & 25.16 \\
\hline \multirow{3}{*}{8} & $0-20$ & 1.12 & 34.60 & 0.78 & 234.00 & 29.70 & 7.54 \\
\hline & $20-55$ & 1.04 & 30.10 & 1.20 & 225.00 & 27.72 & 27.16 \\
\hline & $55-100$ & & & & & 21.78 & 23.30 \\
\hline \multirow{3}{*}{9} & $0-25$ & 1.86 & 34.30 & 1.12 & 144.30 & 33.43 & 20.56 \\
\hline & $25-65$ & 1.46 & 36.10 & 3.10 & 146.50 & 36.22 & 20.35 \\
\hline & $65-100$ & & & & & 16.20 & 12.28 \\
\hline \multirow{3}{*}{10} & $0-20$ & 1.65 & 38.80 & 2.37 & 226.20 & 38.47 & 19.07 \\
\hline & $20-60$ & 1.05 & 41.50 & 2.40 & 231.50 & 30.31 & 11.06 \\
\hline & $60-100$ & & & & & 47.22 & 20.21 \\
\hline \multirow{3}{*}{11} & $0-20$ & 1.50 & 44.50 & 1.34 & 163.80 & 29.70 & 19.30 \\
\hline & $20-55$ & 1.20 & 38.60 & 1.61 & 170.50 & 25.74 & 21.36 \\
\hline & $55-100$ & & & & & 26.00 & 15.61 \\
\hline \multirow{3}{*}{12} & $0-20$ & 2.08 & 16.60 & 0.58 & 156.40 & 54.96 & 16.44 \\
\hline & $20-55$ & 1.70 & 18.50 & 1.15 & 161.30 & 43.96 & 23.24 \\
\hline & $55-100$ & & & & & 43.96 & 12.74 \\
\hline
\end{tabular}


Table 3. Cont

\begin{tabular}{|c|c|c|c|c|c|c|c|}
\hline \multirow{2}{*}{ P. no. } & \multirow{2}{*}{$\begin{array}{c}\text { Depth } \\
\text { (cm) }\end{array}$} & \multirow{2}{*}{ OM \% } & \multicolumn{3}{|c|}{ Available (ppm) } & \multicolumn{2}{|c|}{ Exch., meq/100g } \\
\hline & & & $\mathbf{N}$ & $\mathbf{P}$ & $\mathbf{K}^{+}$ & $\mathrm{Ca}^{++}$ & $\mathrm{Mg}++$ \\
\hline \multirow{3}{*}{13} & $0-30$ & 1.90 & 83.10 & 2.19 & 140.90 & 39.85 & 23.18 \\
\hline & $30-60$ & 1.20 & 75.80 & 2.20 & 150.30 & 26.16 & 17.36 \\
\hline & $60-100$ & & & & & 28.08 & 17.04 \\
\hline \multirow{3}{*}{14} & $0-20$ & 1.90 & 76.40 & 1.19 & 120.90 & 43.96 & 19.04 \\
\hline & $20-60$ & 0.95 & 73.10 & 2.14 & 126.10 & 43.96 & 21.14 \\
\hline & $60-90$ & & & & & 26.38 & 38.72 \\
\hline \multirow{3}{*}{15} & $0-20$ & 1.80 & 87.20 & 0.85 & 187.20 & 48.36 & 25.14 \\
\hline & $20-50$ & 1.20 & 81.40 & 1.10 & 191.40 & 48.36 & 25.14 \\
\hline & $50-90$ & & & & & 21.00 & 12.01 \\
\hline \multirow{3}{*}{16} & $0-30$ & 1.24 & 147.30 & 1.25 & 183.30 & 32.09 & 22.39 \\
\hline & $30-60$ & 1.05 & 136.10 & 1.80 & 191.50 & 25.75 & 9.13 \\
\hline & $60-100$ & & & & & 14.30 & 8.43 \\
\hline \multirow{3}{*}{17} & $0-20$ & 2.00 & 37.00 & 0.60 & 144.30 & 49.07 & 25.33 \\
\hline & $20-60$ & 1.50 & 35.10 & 1.20 & 150.60 & 43.16 & 35.64 \\
\hline & $60-100$ & & & & & 47.25 & 12.16 \\
\hline \multirow{3}{*}{18} & $0-35$ & 1.70 & 82.00 & 1.73 & 245.70 & 29.00 & 17.64 \\
\hline & $35-75$ & 1.30 & 68.40 & 1.90 & 255.20 & 29.85 & 15.67 \\
\hline & $75-100$ & & & & & 26.85 & 17.74 \\
\hline \multirow{3}{*}{19} & $0-20$ & 2.09 & 72.20 & 0.71 & 206.70 & 32.98 & 25.82 \\
\hline & $20-50$ & 1.60 & 70.50 & 0.91 & 186.70 & 26.38 & 36.62 \\
\hline & 50-100 & & & & & 32.96 & 27.94 \\
\hline \multirow{3}{*}{20} & $0-20$ & 1.60 & 61.20 & 0.43 & 156.00 & 29.70 & 17.34 \\
\hline & $20-60$ & 1.20 & 52.80 & 1.60 & 140.80 & 33.66 & 21.22 \\
\hline & $60-90$ & & & & & 31.68 & 25.16 \\
\hline \multirow{3}{*}{21} & $0-20$ & 1.80 & 22.90 & 4.45 & 105.30 & 29.70 & 25.18 \\
\hline & $20-50$ & 1.43 & 24.30 & 3.60 & 115.40 & 38.13 & 21.46 \\
\hline & $50-80$ & & & & & 39.58 & 23.18 \\
\hline \multirow{3}{*}{22} & $0-20$ & 2.05 & 26.40 & 4.16 & 120.60 & 28.78 & 18.5 \\
\hline & $20-55$ & 1.70 & 25.30 & 3.99 & 123.20 & 24.18 & 17.94 \\
\hline & $55-100$ & & & & & 28.98 & 15.22 \\
\hline \multirow{3}{*}{23} & $0-15$ & 1.95 & 8.20 & 1.23 & 206.70 & 22.87 & 27.74 \\
\hline & $15-45$ & 1.60 & 12.60 & 3.60 & 196.80 & 22.54 & 25.32 \\
\hline & $45-100$ & & & & & 17.82 & 20.29 \\
\hline \multirow{2}{*}{24} & $0-15$ & 1.60 & 15.60 & 2.56 & 168.90 & 17.56 & 17.07 \\
\hline & $15-40$ & 1.00 & 16.70 & 3.12 & 171.60 & 25.06 & 14.23 \\
\hline \multirow{3}{*}{25} & $0-20$ & 1.70 & 14.40 & 2.91 & 226.20 & 19.58 & 1418 \\
\hline & $20-50$ & 0.90 & 16.20 & 3.01 & 190.20 & 16.16 & 17.36 \\
\hline & $50-80$ & & & & & 18.08 & 14.04 \\
\hline \multirow{3}{*}{26} & $0-15$ & 2.05 & 37.00 & 1.07 & 265.20 & 20.92 & 13.24 \\
\hline & $15-60$ & 1.70 & 38.00 & 2.05 & 220.40 & 24.56 & 13.04 \\
\hline & $60-100$ & & & & & 15.56 & 19.74 \\
\hline 27 & $0-20$ & 2.20 & 18.50 & 0.95 & 187.20 & 22.36 & 12.71 \\
\hline
\end{tabular}


Table 4. Land capability classes

\begin{tabular}{|c|c|c|c|c|c|c|c|}
\hline P. no. & $\begin{array}{c}\text { Soil } \\
\text { Index }\end{array}$ & W. index & F. index & E. index & $\begin{array}{l}\text { Final } \\
\text { Index }\end{array}$ & $\begin{array}{l}\text { Productivit } \\
\text { y Class }\end{array}$ & Constrains \\
\hline 1 & fair & Excellent & poor & excellent & fair & 3 & GWD,GWS,N,P,K,OM. \\
\hline 2 & fair & Excellent & fair & Excellent & good & 2 & GWS,,OM,P, \\
\hline 3 & fair & Excellent & poor & good & fair & 3 & GWD.GWS,OM,N,P,K. \\
\hline 4 & fair & Excellent & poor & good & fair & 3 & GWD,GWS,ECe,OM, ,P,K. \\
\hline 5 & fair & Excellent & fair & good & good & 2 & GWD,GWS,OM,P,K. \\
\hline 6 & fair & Excellent & poor & good & fair & 3 & GWD,GWS,OM,P. \\
\hline 7 & fair & Excellent & fair & good & good & 2 & GWS,ECe,OM,P. \\
\hline 8 & fair & Excellent & poor & good & fair & 3 & GWS,OM,P. \\
\hline 9 & fair & Excellent & fair & good & fair & 3 & GWS,OM,P. \\
\hline 10 & fair & Excellent & fair & good & good & 2 & GWS,OM,P. \\
\hline 11 & fair & Excellent & poor & good & fair & 3 & $\mathrm{GWD}, \mathrm{GWS}, \mathrm{K}_{\mathrm{s}}, \mathrm{OM}, \mathrm{P}, \mathrm{K}$. \\
\hline 12 & fair & Excellent & poor & good & fair & 3 & $\mathrm{GWD}, \mathrm{GWS}, \mathrm{K}_{\mathrm{s}}, \mathrm{OM}, \mathrm{N}, \mathrm{P}, \mathrm{K}$. \\
\hline 13 & poor & Excellent & poor & good & fair & 3 & GWD,GWS,K $, \mathrm{OM}, \mathrm{P}, \mathrm{K}$. \\
\hline 14 & fair & Excellent & poor & good & fair & 3 & GWD,GWS,OM,P,K. \\
\hline 15 & fair & Excellent & poor & good & fair & 3 & GWD,GWS,OM,P,K. \\
\hline 16 & fair & Excellent & poor & good & fair & 3 & soil struct.,GWS,OM,P,K. \\
\hline 17 & fair & Excellent & poor & good & fair & 3 & GWD,GWS,OM,P,K. \\
\hline 18 & good & Excellent & poor & good & fair & 3 & GWS,OM,P,K.. \\
\hline 19 & fair & Excellent & fair & good & fair & 3 & GWS,OM,P. \\
\hline 20 & fair & Excellent & poor & good & fair & 3 & $\begin{array}{c}\text { GWD,GWS,OM,P,K,agronom } \\
\text {. Processes }\end{array}$ \\
\hline 21 & fair & Excellent & poor & good & fair & 3 & GWD,GWS,OM,P,K \\
\hline 22 & fair & Excellent & fair & good & fair & 3 & GWD,GWS,OM,P,K. \\
\hline 23 & fair & Excellent & poor & good & fair & 3 & GWD,GWS,OM,N,P. \\
\hline 24 & fair & Excellent & poor & good & fair & 3 & GWD,GWS,OM,N,P. \\
\hline 25 & fair & Excellent & poor & good & fair & 3 & GWS,OM,P. \\
\hline 26 & fair & Excellent & poor & good & fair & 3 & $\begin{array}{c}\text { GWS,OM,N,P,K,agronom. } \\
\text { Processes }\end{array}$ \\
\hline 27 & fair & Excellent & poor & good & fair & 3 & GWD,GWS,OM,P,K. \\
\hline
\end{tabular}

Table 5. Climatic data during the period 2000-2008

\begin{tabular}{|c|c|c|c|c|c|c|c|}
\hline \multirow{2}{*}{ Months } & \multicolumn{3}{|c|}{ Temperature ${ }^{\circ} \mathrm{C}$} & \multirow{2}{*}{$\begin{array}{c}\text { Rain- } \\
\text { fall }\end{array}$} & \multirow{2}{*}{$\begin{array}{l}\text { Evaporat. } \\
\text { mm/month }\end{array}$} & \multirow{2}{*}{$\begin{array}{l}\text { Relative } \\
\text { Humidity }\end{array}$} & \multirow{2}{*}{$\frac{\text { Wind Speed }}{\mathrm{m} / \mathrm{sec}}$} \\
\hline & Max. & Min. & Mean & & & & \\
\hline January & 18.42 & 6.5 & 12.46 & 13.6 & 61 & 80 & 1.29 \\
\hline February & 19.9 & 7 & 13.45 & 12.8 & 66 & 78 & 1.37 \\
\hline March & 22.7 & 8.6 & 15.65 & 5.91 & 75.01 & 76 & 1.7 \\
\hline April & 26.86 & 10.84 & 18.85 & 2.78 & 90.4 & 69 & 1.41 \\
\hline May & 30.2 & 14.5 & 22.35 & 0 & 107.4 & 65 & 1.2 \\
\hline June & 32.1 & 17.8 & 24.95 & 0 & 119.5 & 64 & 1.1 \\
\hline July & 33.8 & 19.86 & 26.48 & 0.2 & 127.6 & 74 & 1 \\
\hline August & 31.5 & 19.6 & 26.2 & 0.4 & 126 & 76 & 1 \\
\hline September & 28.9 & 18.6 & 25.05 & 0.9 & 119.9 & 75 & 1.2 \\
\hline October & 24.8 & 15.7 & 22.3 & 3.5 & 107.5 & 75 & 1 \\
\hline November & 24.8 & 12.5 & 18.65 & 6.25 & 90 & 77 & 1.02 \\
\hline
\end{tabular}




\begin{tabular}{|c|c|c|c|c|c|c|c|c|c|c|}
\hline \multicolumn{2}{|c|}{ December } & 20.66 & 8.5 & 14.58 & \multicolumn{2}{|c|}{12.95} & \multirow{2}{*}{$\begin{array}{c}69.9 \\
\begin{array}{c}\text { Potato } \\
\%\end{array} \\
\end{array}$} & \multicolumn{2}{|c|}{81} & \multirow{2}{*}{ 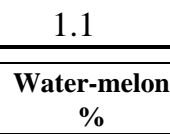 } \\
\hline P. no. & $\begin{array}{c}\text { Alfalfa } \\
\%\end{array}$ & & $\begin{array}{c}\text { Sorghum } \\
\%\end{array}$ & $\begin{array}{c}\text { Onion } \\
\%\end{array}$ & $\begin{array}{c}\text { Cabbage } \\
\%\end{array}$ & $\begin{array}{c}\text { Pea } \\
\%\end{array}$ & & $\begin{array}{c}\text { Pepper } \\
\%\end{array}$ & $\begin{array}{c}\text { Tomato } \\
\%\end{array}$ & \\
\hline 1 & 79.80 & & 85.96 & 37.82 & 86.23 & 78.07 & 29.79 & 39.63 & 48.53 & 40.91 \\
\hline 2 & 35.31 & & 85.89 & 30.50 & 90.28 & 73.56 & 69.43 & 37.69 & 46.41 & 36.03 \\
\hline 3 & 80.23 & & 84.41 & 38.13 & 88.73 & 70.40 & 31.75 & 38.75 & 45.69 & 37.04 \\
\hline 4 & 82.29 & & 83.29 & 33.35 & 83.80 & 75.39 & 29.99 & 33.89 & 46.13 & 34.99 \\
\hline 5 & 35.49 & & 88.04 & 31.13 & 92.54 & 85.96 & 72.69 & 40.41 & 47.57 & 38.63 \\
\hline 6 & 35.49 & & 88.04 & 29.40 & 92.54 & 85.96 & 72.69 & 39.85 & 35.16 & 38.63 \\
\hline 7 & 70.20 & & 747.23 & 20.18 & 73.67 & 69.54 & 25.58 & 37.85 & 36.16 & 31.35 \\
\hline 8 & 84.20 & & 87.66 & 37.86 & 88.08 & 85.59 & 32.96 & 39.72 & 34.34 & 38.47 \\
\hline 9 & 78.67 & & 83.39 & 33.34 & 83.79 & 75.78 & 27.76 & 33.88 & 42.64 & 32.39 \\
\hline 10 & 79.28 & & 82.55 & 32.76 & 82.94 & 79.70 & 29.56 & 32.80 & 35.40 & 32.06 \\
\hline 11 & 87.22 & & 78.68 & 38.14 & 82.70 & 71.12 & 31.35 & 33.50 & 40.10 & 34.52 \\
\hline 12 & 90.82 & & 33.44 & 36.10 & 33.60 & 77.12 & 28.60 & 34.12 & 36.10 & 33.20 \\
\hline 13 & 87.62 & & 81.82 & 37.91 & 82.21 & 76.36 & 32.61 & 35.44 & 45.60 & 35.90 \\
\hline 14 & 80.40 & & 88.28 & 36.44 & 88.70 & 76.29 & 31.74 & 38.23 & 45.60 & 39.46 \\
\hline 15 & 82.52 & & 89.93 & 37.55 & 90.36 & 87.80 & 32.70 & 40.74 & 47.96 & 39.46 \\
\hline 16 & 70.29 & & 87.83 & 31.16 & 91.85 & 78.99 & 29.60 & 33.67 & 42.81 & 34.77 \\
\hline 17 & 82.73 & & 85.07 & 35.52 & 89.91 & 83.05 & 31.40 & 39.05 & 40.21 & 37.33 \\
\hline 18 & 74.30 & & 92.77 & 33.60 & 96.24 & 90.57 & 32.90 & 42.03 & 49.40 & 40.71 \\
\hline 19 & 82.69 & & 82.88 & 33.14 & 83.28 & 74.92 & 28.87 & 33.67 & 42.81 & 34.77 \\
\hline 20 & 35.04 & & 87.17 & 33.56 & 91.62 & 78.79 & 70.46 & 38.25 & 47.1 & 36.56 \\
\hline 21 & 80.53 & & 81.19 & 35.06 & 85.34 & 79.27 & 30.54 & 37.27 & 43.87 & 35.63 \\
\hline 22 & 85.53 & & 89.46 & 29.71 & 92.81 & 87.34 & 33.59 & 41.85 & 47.71 & 40.53 \\
\hline 23 & 24.69 & & 79.11 & 38.10 & 89.81 & 84.51 & 62.24 & 37.13 & 45.72 & 34.38 \\
\hline 24 & 82.73 & & 91.81 & 35.77 & 92.25 & 79.33 & 32.59 & 39.76 & 47.42 & 40.29 \\
\hline 25 & 70.30 & & 90.37 & 37.78 & 93.75 & 88.23 & 23.83 & 41.87 & 47.73 & 40.55 \\
\hline 26 & 26.43 & & 91.89 & 22.30 & 95.33 & 89.71 & 24.26 & 42.57 & 48.53 & 41.23 \\
\hline 27 & 82.60 & & 91.91 & 38.44 & 92.35 & 86.91 & 35.85 & 41.82 & 47.68 & 41.82 \\
\hline $\mathrm{Wi}$ & & 19.56 & 9.8 & 16.18 & & & 64.75 & & & 1.25 \\
\hline Sun & & 31.09 & 16.87 & 23.98 & & & 115.13 & & & 1.15 \\
\hline
\end{tabular}

Table 6a. Land suitability indices for different vegetables and forage crops

Table 6b . Land suitability indices for different field crops 
Table 6c. Land suitability indices for different fruit trees

\begin{tabular}{|c|c|c|c|c|c|c|c|c|c|}
\hline P. no & \multicolumn{2}{|c|}{$\begin{array}{c}\text { Citrus } \\
\% \\
\end{array}$} & $\begin{array}{c}\text { Banana } \\
\% \\
\end{array}$ & $\begin{array}{c}\text { Olive } \\
\% \\
\end{array}$ & \multicolumn{2}{|l|}{$\begin{array}{c}\text { Pear } \\
\%\end{array}$} & \multicolumn{2}{|l|}{$\begin{array}{c}\text { Date Palm } \\
\% \\
\end{array}$} & $\begin{array}{c}\text { Fig } \\
\%\end{array}$ \\
\hline 1 & \multicolumn{2}{|c|}{29.33} & 67.39 & 42.71 & \multicolumn{2}{|l|}{66.79} & \multicolumn{2}{|l|}{33.06} & 31.60 \\
\hline 2 & \multicolumn{2}{|c|}{73.21} & 31.09 & 37.53 & \multicolumn{2}{|l|}{30.69} & \multicolumn{2}{|l|}{32.80} & 32.40 \\
\hline 3 & \multicolumn{2}{|c|}{33.26} & 74.49 & 36.89 & \multicolumn{2}{|l|}{73.52} & \multicolumn{2}{|l|}{34.57} & 32.00 \\
\hline 4 & \multicolumn{2}{|c|}{28.44} & 64.49 & 35.23 & \multicolumn{2}{|l|}{63.65} & \multicolumn{2}{|l|}{31.98} & 30.57 \\
\hline 5 & \multicolumn{2}{|c|}{74.46} & 31.42 & 37.20 & \multicolumn{2}{|l|}{70.83} & \multicolumn{2}{|l|}{76.51} & 70.83 \\
\hline 6 & \multicolumn{2}{|c|}{74.46} & 31.40 & 37.30 & 68.18 & & 73.54 & & 71.60 \\
\hline 7 & \multicolumn{2}{|c|}{56.40} & 31.21 & 32.33 & 64.20 & & 31.21 & & 27.63 \\
\hline 8 & & & 78.58 & 38.31 & 77.48 & & 34.32 & & 33.24 \\
\hline 9 & & & 66.71 & 35.29 & 65.85 & & 33.07 & & 30.62 \\
\hline 10 & & & 64.99 & 34.87 & 64.15 & & 31.65 & & 31.20 \\
\hline 11 & & & 73.82 & 33.24 & 72.87 & & 33.00 & & 32.16 \\
\hline 12 & & & 76.87 & 36.01 & 75.87 & & 32.46 & & 31.15 \\
\hline 13 & & & 77.58 & 34.57 & 76.57 & & 32.81 & & 31.78 \\
\hline 14 & & & 69.74 & 35.20 & 67.11 & & 30.54 & & 30.54 \\
\hline 15 & & & 72.14 & 35.86 & 71.20 & & 31.06 & & 30.60 \\
\hline 16 & & & 71.25 & 37.54 & 78.68 & & 79.02 & & 73.16 \\
\hline 17 & & & 73.25 & 35.94 & 72.30 & & 32.56 & & 32.16 \\
\hline 18 & & & 32.67 & 39.19 & 32.25 & & 77.06 & & 74.64 \\
\hline 19 & & & 62.76 & 35.02 & 63.96 & & 31.78 & & 30.38 \\
\hline 20 & & & 48.90 & 34.76 & 74.08 & & 70.92 & & 67.80 \\
\hline 21 & & & 29.64 & 33.26 & 29.26 & & 59.61 & & 60.42 \\
\hline Profileno. & Wheat? & 0\$3arley & Sunflguxer & Sugerbyets & Cott90.90 & Maize & Fäbatgen & Rice & 29592a \\
\hline $1^{23}$ & $\frac{\%}{87.24}$ & $16 \frac{\%}{87.24}$ & $\begin{array}{r}48 \% .12 \\
92.84\end{array}$ & 35.60 & $\frac{\%_{23} .42}{90.21}$ & $\begin{array}{c}\% \\
79.59\end{array}$ & $\frac{40 \%}{71.41}$ & $\begin{array}{c}\% \\
81.03\end{array}$ & 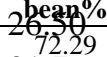 \\
\hline $2^{24}$ & 37.23 & $10_{37.23}$ & 32.76 & 35.8579 & $90.28^{2} .30$ & 76.58 & $379: 80$ & 36.23 & 31.7 .88 \\
\hline 325 & 85.262 & 8685.26 & Q4.BO & 8477509 & $88.7 \not 71.91$ & 81.76 & 38.52 & 85.67 & 22.6 .80 \\
\hline${ }^{4} 26$ & 86.26 & $26^{86.26}$ & 86.950 & 8377471 & 85.593 .82 & 77.22 & $32: 18$ & 80.25 & $226.43^{5}$ \\
\hline $\begin{array}{l}5 \\
627\end{array}$ & $\begin{array}{l}37.20 \\
37.20\end{array}$ & $95_{37.20}^{37.20}$ & $\begin{array}{r}92.84 \\
69.89 \\
\end{array}$ & $\begin{array}{r}3588 \\
338 \\
35800\end{array}$ & $\begin{array}{r}90.35 \\
90.357 .97\end{array}$ & $\begin{array}{r}85.27 \\
85.27\end{array}$ & $\begin{array}{r}52.100 \\
3.39 \\
3.84 \\
\end{array}$ & $\begin{array}{r}36.20 \\
36.21\end{array}$ & $\begin{array}{r}72.45 \\
33,440 \\
\end{array}$ \\
\hline 7 & 75.73 & 79.22 & 79.16 & 74.72 & 82.17 & $71 . .74$ & 20.56 & 72.65 & 21.87 \\
\hline 8 & 85.90 & 85.90 & 90.07 & 83.73 & 87.65 & 71.84 & 68.74 & 86.31 & 81.64 \\
\hline 9 & 83.56 & 86.27 & 90.07 & 83.73 & 87.65 & 71.84 & 68.74 & 80.26 & 69.79 \\
\hline 10 & 86.94 & 86.94 & 87.04 & 82.88 & 84.71 & 70.76 & 68.04 & 80.88 & 64.99 \\
\hline 11 & 91.43 & 91.43 & 82.96 & 88.67 & 80.74 & 76.20 & 64.86 & 88.98 & 64.29 \\
\hline 12 & 95.21 & 95.21 & 35.26 & 90.46 & 34.32 & 30.96 & 29.03 & 92.65 & 29.03 \\
\hline 13 & 91.85 & 91.85 & 82.48 & 88.15 & 82.87 & 79.25 & 72.85 & 89.39 & 72.21 \\
\hline 14 & 85.29 & 85.29 & 86.03 & 84.79 & 86.45 & 81.74 & 74.05 & 83.00 & 72.77 \\
\hline 15 & 86.50 & 86.50 & 87.64 & 83.45 & 88.06 & 87.10 & 83.76 & 37.38 & 80.27 \\
\hline 16 & 35.80 & 35.80 & 92.14 & 36.48 & 89.67 & 80.90 & 72.03 & 79.20 & 72.03 \\
\hline 17 & 86.72 & 86.72 & 89.70 & 82.58 & 87.30 & 80.20 & 75.74 & 37.47 & 75.07 \\
\hline 18 & 36.98 & 36.98 & 93.51 & 85.65 & 93.96 & 89.85 & 86.40 & 37.16 & 85.35 \\
\hline 19 & 86.69 & 86.69 & 87.40 & 83.22 & 85.06 & 76.74 & 68.32 & 80.65 & 67.73 \\
\hline 20 & 36.73 & 36.73 & 88.86 & 36.39 & 86.84 & 80.71 & 71.85 & 35.75 & 68.86 \\
\hline 21 & 84.42 & 84.42 & 80.02 & 81.52 & 77.87 & 78.63 & 72.28 & 82.16 & 66.97 \\
\hline 22 & 86.84 & 86.84 & 87.18 & 83.01 & 87.60 & 89.46 & 83.32 & 35.52 & 79.89 \\
\hline 23 & 25.07 & 25.07 & 79.11 & 24.69 & 75.98 & 76.62 & 73.67 & 25.19 & 66.21 \\
\hline 24 & 86.72 & 86.72 & 92.54 & 83.18 & 92.99 & 88.92 & 75.86 & 36.29 & 75.02 \\
\hline 25 & 81.41 & 81.41 & 90.37 & 81.34 & 90.80 & 90.37 & 84.16 & 26.55 & 82.67 \\
\hline 26 & 26.83 & 26.83 & 91.89 & 26.00 & 92.23 & 91.89 & 85.58 & 26.96 & 84.51 \\
\hline 27 & 83.87 & 83.87 & 89.73 & 85.44 & 90.16 & 91.91 & 85.60 & 84.27 & 84.85 \\
\hline
\end{tabular}


The data (Tables $6 a, 6 b, 6 c$ ) revealed that, those soils are highly suitable for Wheat, Barley, and Sugarbeet, Sunflower, Rice, Sorghum, Cotton and Alfalfa. While it was suitable for Fababean, Soybean, Pear and Banana. It could be used for all crops expect Pepper, Olive, Fig and Peanut.

Maps (3, 4, 5 and 6) show the land suitability for some selected crops for Rewena area and indicate that; the most of the area around $85 \%$ was highly suitable for alfalfa, cabbage, wheat and sugar beet; where the highly suitable index $(\mathrm{S} 1+\mathrm{S} 2)$ were 88.56, 85.53, 81.89 and $77.28 \%$ respectively.

On the other hand very small area around $4 \%$ was unsuitable for mentioned crops.

Also, table (6c) indicate that the most of the area was unsuitable for citrus cultivation.

Concerning banana crop; map (Fig. 7) show that about $56.5 \%$ from total area were highly suitable while $4 \%$ were unsuitable.

\section{Recommendation:}

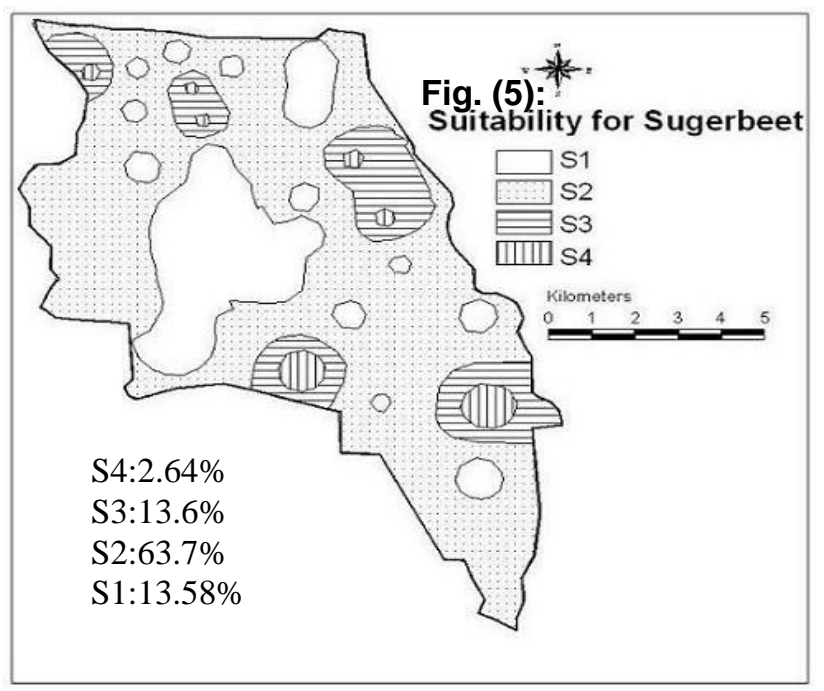

For maximizing the soil productivity of Rewena area it is recommended that;

- Increasing the drainage efficiency, through periodical maintenances of title drainage system.

- Carrying out sub soiling processes to remove salts and /or hard pans which may exist in such heavy clay soil.

- Deep plowing should be carried out to prevent the upward movement of saline ground water to the soil surface through capillary rise.

- Application of organic matter and soil amendments, to improve physical soil properties and nutrient statues.

- Proper fertilization (type, time, amount and place of application) must be followed under the saline soil condition.
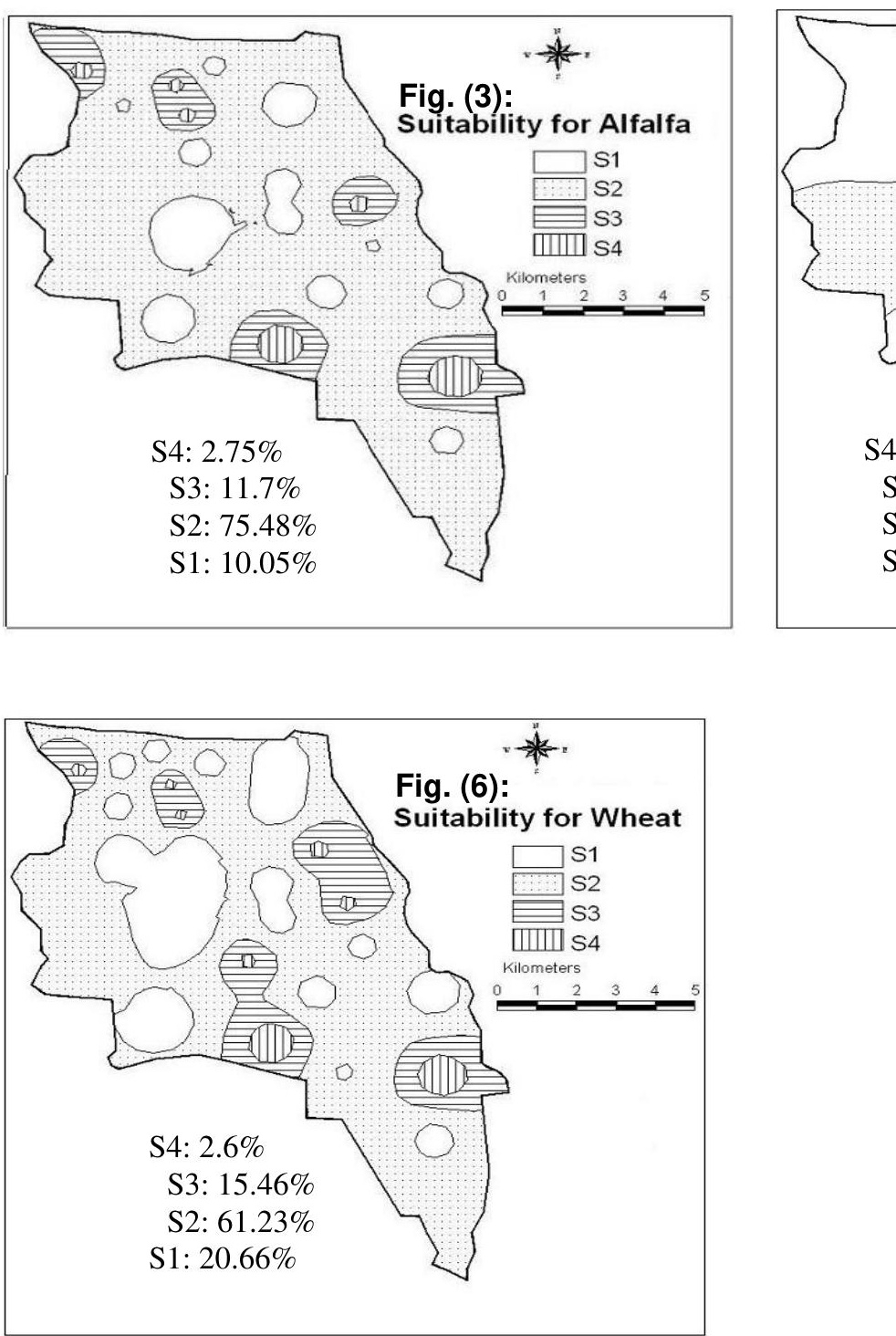


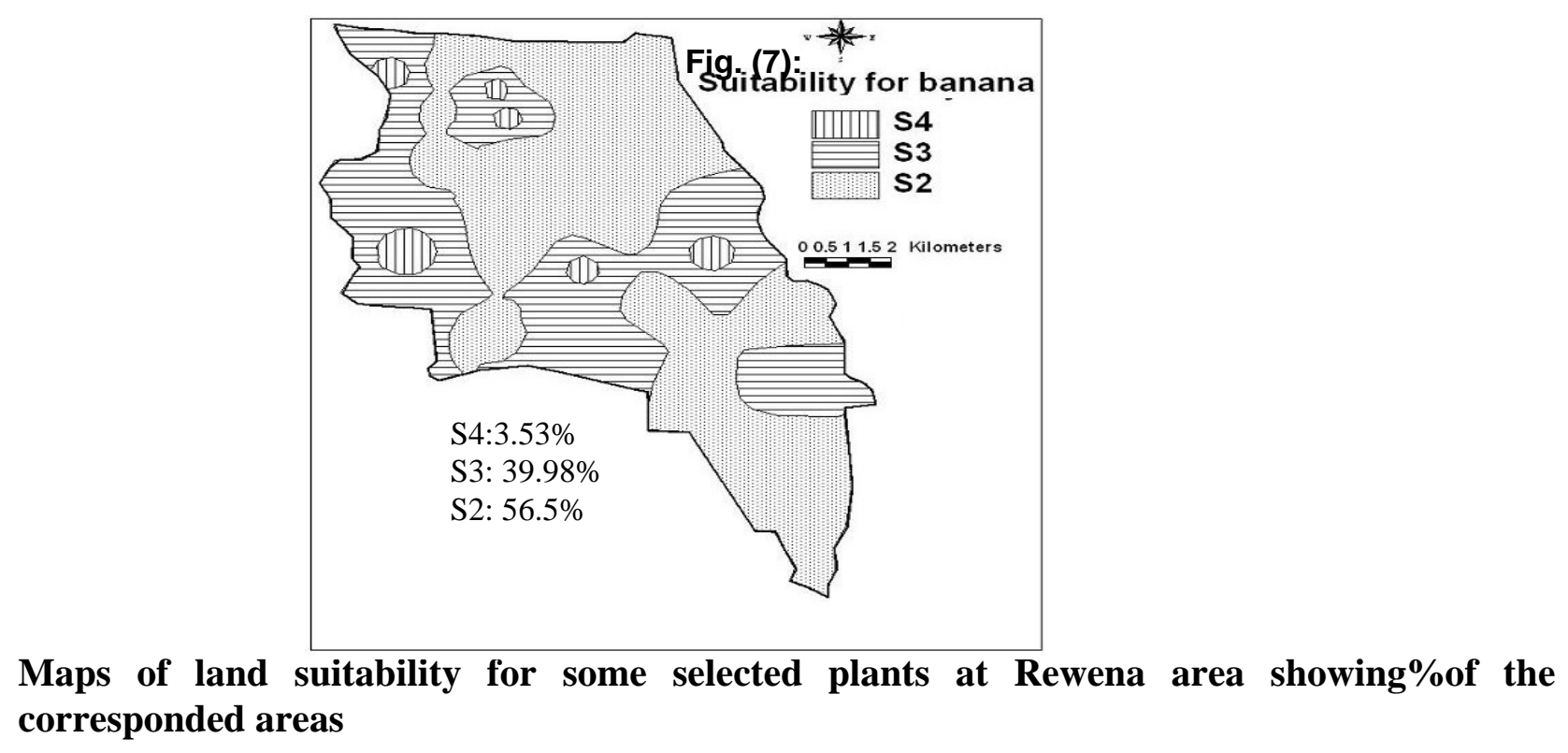

\section{RE FERENCES}

Cottenie, A.M. Nerloo, G. Velghe and L. Kiekene (1982). Biological analytical aspects of soil pollution. Lab. Of analytical Agro. State Univ. Ghent- Belgium

EL-Fayoumy, M.F. (1989). New approach of land evaluation of some Egyptian regions. Ph.D. Thesis, fac. of Agric .univ. of Alexandria.

FAO (1976). A framework for land evaluation. Solis Bull. No32, FAO, Rome.

Fayed .R .I .M (2003). Impact of land management practices on soil , El-Bostan Region - Egypt. ph. D thesis- Fac. Agric Alex univ.

FAO (2001). Agriculture trade and Rual Department in the Middle East and North Africa. Recent Department and prospects. The World Bank international economics department.

Fayed, R.I.M. (2003). Impact of land management practices on soil, El-Bostan Region - Egypt. ph. D. thesis- Fac. Agric Alex. univ.

GARPAD, 1997). General Authority for Reclamation projects and development. Strategy for horizontal land reclamation in Egypt until year 2017, Ministry of Agriculture, Egypt. (In Arabic).

Higab, I.A. (2005). An evaluation of some soils of south ElBorolous lake Area. PhD thesis, Fac. Agric Al-Azhar univ.

Ismail, H.A., S.M. Marei and M.E. El-Fayoumy (1994) A modify approach for land evaluation under arid condition. I. Basis and Computer program. J. Agric. Sci., Mansoura Univ., 19 (10): 3483 - 3495.

Ismail H.A., I.M..Morsy, E.M. EL-Zahaby and F.S. El-Nagar (2001). A Developed Expert System for land use planning by coupling Land Information System (LIS) and Modeling. Alex. Agric. Res. 46(3):141-154.
Jackson, M.L. (1973). Soil chemical analysis. Advanced course Ed.2. A Manual of methods useful for instruction and research in soil chemistry, physical chemistry of soil, soil fertility and soil genesis. Revised from Original Edition (1955).

Klute, A. (ed.) (1986). Methods of soil analysis (part1). Physical and mineralogical methods ( ${ }^{\text {nd }}$ Ed.). American society of agronomy. Inc. Soil Sci. of Agron. Inc. Madison. Wisconsin, USA.

Madkour, H.E.A., A.M.M. Hamouda and S.A. Moussa (1999). Hydrulic conductivity and structure factor of alluvial soils as affected by some soil properties. J. Agric. Sci Mansoura Univ,, 24(8) : 4249-4258 .

Marei, S.M., H.A. Ismail and H.Meshref (1987). A. computer program for land evaluation in Egypt. J. Agric. Sci. Mansoura Univ. 12 (4):1427-1447.

Morsy, I.M (1994). A model relating soil properties and variability to land capability and suitability for crops in arid irrigated region .PhD thesis. fac. of Agric ,Univ. of Alexandria.

Nasr EL-Din, I.E.I. (2001). Evaluation of some different soils from Kafer EL-Sheikh under different crop patterns .Ph.D.Thesis,fac.of Agric. Kafer El-Sheikh, Tanta Univ.

Olsen, S, R; C. V. Cole; F. S. Watambe and L. A. Dean ( 1954 ). Estimated of available phosphorus in soil by sodium bicarbonate. U. S. Dept. Agric. Cire. 939.

Page, A.L. (1982). Methods of soil analysis part2: Chemical and microbiological properties ( $2^{\text {nd }}$ ed.) Amer. Soc. Agron. In soil Sci. soc. Amer. Inc. Madison, Wisconsin, UAA Chapter12:pp. 199-223.

Piper, C.S.(1950). Soil and Plant Analysis. Inter. Sci. Public. Inc. New York. 
Richards, R.L. (ed.) (1954). Diagnosis and improvement of saline and alkali soils. Agriculture Hand Book No. 60, U.S Govt. Printing Office, Washington, USA.

Riquier, j., D.L .Bramao and J.P. Cornet (1970). A new system of soil appraisal in terms of actual and potential productivity .FAO soil resource, development and conservation service, land and water development Division, FAO, Rome.

Sys, C. and Verheye, W. (1972). Principals of land classification in arid and semiarid regions. State Univ. Ghent, Belgium.
Sys, C. (1979). Evaluation of the physical environment for irrigation in terms of land characteristics and land.

Van Beers, W.F.J. (1970). The auger hole method. Bull. No 8, 11. RI Wageningen, the Netherlands.

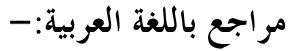

محمد أحمد عبد المطلب وسيف الدين أحمد حسـين (910 (1) ). تقيسيم الأراضي الزراعية. كلية الزراعة - جامعة الأزهر.

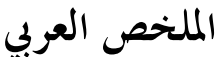

\section{تقييم الأراضي المروية القديمة (أراضى ترعه روينه) في محافظة كفر الشيخ منطقة شمال الدلتا}

هجت عبد القوى زامل، محمد عبد الله احمد عبد الله، جمال محمد عبد السلام ومحمد إسماعيل الشهاوي

التالي:

الشعير > القمح > بنجر السكر > عباد الشمس > القطــن

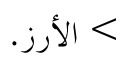

ولتعظيم الأنتاجيه في هذه الأراضي موضع الدراسة بيجــب

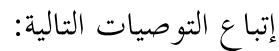

زيادة كفاءة الصرف بالصيانة الدورية لنظام الصرف المغطىى

$$
\text { واستخدام و سائل الصرف المساعده }
$$

القيام بعمليات الحرث تحت التربة لإزالة الأمــلاحح الزائـــدة

$$
\text { والطبقات الصماء وبخاصة في الأراضي الطينية الثقيلة. }
$$

الخدمة العميقة لمنع ارتفاع الماء الأرضي مرتفع الملوحة بالخاصية

$$
\text { الشعرية إلى سطح التربة. }
$$

أضافه المادة العضوية ومحسنات التربة لتحسين خواص التربـــة الفزيائيه وحاله المغذيات.

الاهتمام بالتسميد (النوع، و الزمن، الكمية ومكان الإضـــافة)

تم استخدام الخُصائص الكيماوية والطبيعية وموقف المغــذيات

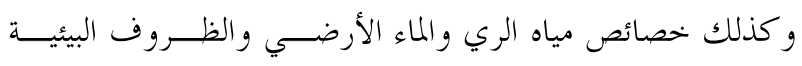
المختلفة كمدخلات لبرنامج ASLE الذي تم استخدامه في حساب

$$
\text { التي يجب إن تضاف في حاله الأراضى الملحية. }
$$


القدرة الإنتاجية للتربة ومدى ملاءمتها لمختلف المحاصيل واوضحت

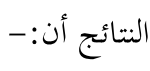

المساحة التي تمت دراستها تقع في الرتب الإنتاجية الآتية: الرتبة

$$
\text { الثانية (جيدة)، و الرتبة الثالثة (متوسطة). }
$$

كانت أهم المحددات لمقدرة الأرض الإنتاجية تتمثل في النخفاض

نفاذية الأرض وارتفاع مستوى الماء الارضى و كذلك ارتفاع ملوحة التربة والماء الارضى وانخفاض محتوى الأرض من المـــادة العضـــــية والمغذيات، خاصة النتروجين والفسفور و البوتاسيوم.

فيما يتعلق بملائمة الأرض لمختلف المحاصيل تلاحظ أن هــــه

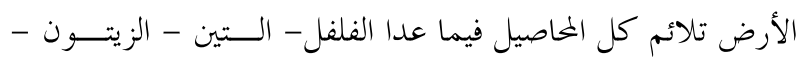

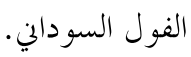

ومن الممكن ترتيب ملائمة التربة للمحاصيل في الترتيب التنازلي 